\title{
A Rare Cause of Obscure Occult Gastrointestinal Bleeding
}

Jefferson。

\author{
Haroon Shahid MD, Jason Korenblit MD, MBA
}

Department of Gastroenterology and Hepatology, Thomas Jefferson University Hospital, Philadelphia, PA, United States

\section{BACKGROUND}

- Obscure gastrointestinal (GI) bleeding is defined as persistent or recurrent GI bleeding after negative evaluations with upper endoscopy and colonoscopy.

- Accounts for approximately 5\% of GI bleeding.

- Obscure GI bleeding can further be classified as being overt or occult.

- Overt GI bleeding is clinically evident, with either hematemesis, hematochezia, or melena.

- Occult GI bleeding manifests as iron deficiency anemia or a positive fecal occult blood test.

- Our case demonstrates a rare cause of obscure occult GI bleeding.

\section{CASE PRESENTATION}

- An 86 year-old man with a history of stroke and atrial fibrillation presented for an outpatient evaluation of anemia. He took rivaroxaban due to his atrial fibrillation. He denied any symptoms other than fatigue. He did not have any overt signs of GI bleeding.

- On labwork, he was found to have a hemoglobin of 9.4 and iron deficiency anemia.

- He underwent an EGD and colonoscopy which revealed mild duodenitis and pan-diverticulosis, respectively.

- A capsule study showed a possible polyp in the proximal to mid-jejunum but no active GI bleeding.

- Antegrade single-balloon enteroscopy was performed, which showed a large villous, pedunculated $8 \mathrm{~cm}$ mass in the proximal jejunum with stigmata of recent bleeding. This appeared to malignant.

- A hot snare was used to completely remove the polyp.

- Histology demonstrated a hamartomatous, PuetzJeghers type polyp which was negative for dysplasia.
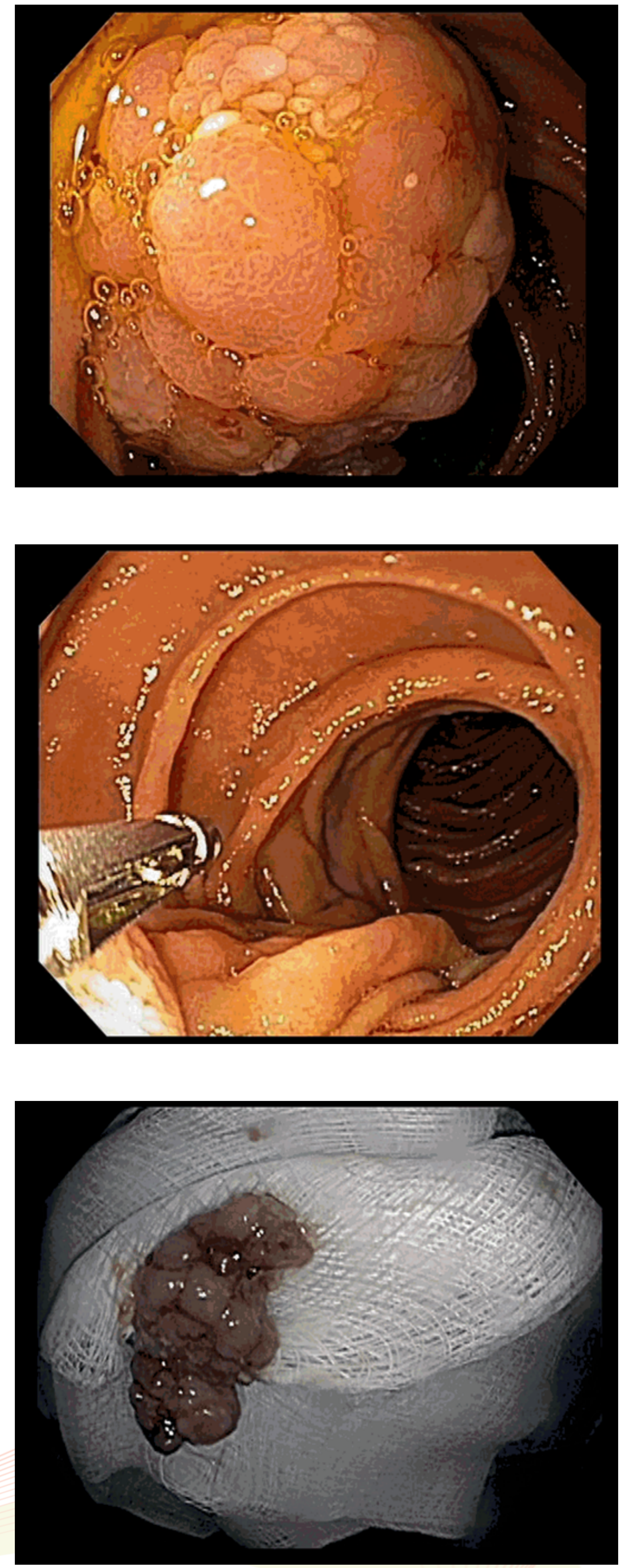

\section{DISCUSSION}

- Solitary Puetz-Jeghers type polyps (PJP) are rare and thought to be distinct from Puetz-Jeghers Syndrome (PJS)

Differs from PJS, because not associated with mucocutaneous pigmentation or a family history of PJS

- Can occur anywhere in the GI tract and their incidence is not known.

- They are oftentimes asymptomatic.

- Rarely can present with obstruction, GI bleeding, or abdominal pain

- Assumed to have a low risk of malignant transformation, unlike GI hamartomas in PuetzJeghers Syndrome.

Experts recommend removing PJPs endoscopically or surgically given the risk for malignant transformation

\section{REFERENCES}

1) Sekino Y, et al. Solitary Peutz-Jeghers type hamartomatous polyps in the duodenum are not always associated with a low risk of cancer: two case reports. Journal of Medical Case Reports 2011, 5:240.

2) Suzuki S, Hirasaki S, Ikeda F, Yumoto E, Yamane H, Matsubara M: Three cases of Solitary Peutz-Jeghers-type hamartomatous polyp in the duodenum. World J Gastroenterol 2008, 14:944-947.

3) Sone $Y$, Nakano S, Takeda I, Kumada T, Kiriyama S, Hisanaga Y: Solitary hamartomatous polyp of Peutz-Jeghers type in the jejunum resected endoscopically. Gastrointest Endosc 2000, 51:620-622.

4) Beggs AD, Latchford AR, Vasen HF, et al. Peutz-Jeghers syndrome: a systematic review and recommendations for management. Gut. 2010;59:975-86

5) Choi, Chang, et al. Solitary Peutz-Jeghers-type appendiceal hamartomatou polyp growing into the terminal ileum. World J Gastroenterol. 2014 April 28; 20(16): 4822-4826.

6) Giardello FM, Trimbath JD. Peutz-Jeghers syndrome and management recommendations. Clinical Gastro Hepatol 2006. Apr;4(4):408-415. 